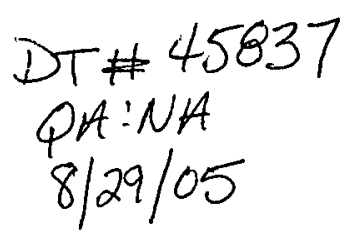

\title{
Water Imbibition into Rock as Affected by Sample Shape, Pore Connectivity, and Antecedent Water Content
}

Robert P. Ewing, Iowa State University, Department of Agronomy, Iowa State University, Ames, IA 50011-1010 and Qinhong (Max) Hu, Lawrence Livermore National Laboratory, PO Box 808, Mail Stop L-231, 7000 East Ave., Livermore, CA 94550

Infiltration is often presumed to follow Philip's equation, $I=s t^{1 / 2}$, where $I$ is cumulative infiltration, $s$ is sorptivity, and $t$ is time. This form of the equation is appropriate for short times, and/or for negligible gravitational effects. For a uniform soil, this equation describes a plot of $\log$ (mass imbibed) versus $\log ($ time), with a slope (imbibition exponent) of $1 / 2$. The equation has also been applied to low-porosity rocks, where the extremely small pores render gravitational forces negligible.

Experiments recently performed on a wide variety of rocks produced imbibition exponents from 0.2 to 0.5 . Many rock types showed initial imbibition proceeding as $I$ $t^{1 / 4}$, then later switched to "normal" $\left(t^{1 / 2}\right)$ behavior. The distance to the wetting front that corresponds to this cross-over behavior was found to be related to the sample shape: tall thin samples are more likely to exhibit the exponent 1/4, and to cross over to 1/2-type behavior later, while short, squat samples are less likely to display the 1/4-type behavior at all. Additionally, the exponents are sensitive to antecedent water content, with initially wetter samples having smaller values.

In this study, we present the experimental data, and provide a consistent and physicallybased explanation using percolation theory. The analogy between imbibition and diffusion is used to model imbibition into samples with low pore connectivity, with the exponents and their crossover behavior emerging from a random walk process. All laboratory phenomena - different exponents, crossover behavior, and effects of sample shape and antecedent water content - are reproduced by the model, with similar patterns across experiment and simulation. We conclude both that diffusion is a useful and powerful conceptual model for understanding imbibition, and also that imbibition experiments, being simpler than diffusion measurements, can be used to examine diffusive behavior in rock.

This work was supported by the United States Department of Energy (DOE), Office of Civilian Radioactive Waste Management (OCRWM), Office of Science and Technology and International (OST\&I). This work was performed under the auspices of the U.S. Department of Energy by University of California, Lawrence Livermore National Laboratory under Contract W-7405-Eng-48 